\title{
The Kibble-Zurek mechanism in a subcritical bifurcation
}

\author{
M A Miranda, D Laroze L $^{1,2}$ and W González-Viñas ${ }^{3,4}$ \\ ${ }^{1}$ Max Planck Institute for Polymer Research, D-55021 Mainz, Germany \\ ${ }^{2}$ Instituto de Alta Investigación, Universidad de Tarapacá, Casilla 7D, Arica, Chile \\ ${ }^{3}$ Department of Physics and Applied Mathematics, University of Navarra, Pamplona, Spain \\ E-mail: montse@alumni.unav.es, laroze@mpip-mainz.mpg.de and wens@unav.es
}

Received 2 February 2013, in final form 12 March 2013

Published 11 September 2013

Online at stacks.iop.org/JPhysCM/25/404208

\begin{abstract}
We present a study of the freezing dynamics of topological defects in a subcritical system by testing the Kibble-Zurek (KZ) mechanism while crossing a tri-stable region in a one-dimensional quintic complex Ginzburg-Landau equation. The critical exponents of the $\mathrm{KZ}$ mechanism and the horizon (KZ-scaling regime) are predicted from the quasistatic study, and are in full accordance with the quenched study. The correlation length, in the KZ freezing regime, is corroborated from the number of topological defects and from the spatial correlation function of the order parameter. Furthermore, we characterize the dynamics to differentiate three out-of-equilibrium regimes: the adiabatic, the impulse and the free relaxation. We show that the impulse regime shares a common temporal domain with a fast exponential increase of the order parameter.
\end{abstract}

(Some figures may appear in colour only in the online journal)

\section{Introduction}

The coexistence of different patterns or states in nature may arise from the subcritical nature of instabilities. The Kibble-Zurek (KZ) mechanism was initially established for second-order phase transitions [1-4] under which many experiments tested its validity in different systems such as liquid crystals [5-7], ${ }^{3} \mathrm{He}[2,8-10],{ }^{4} \mathrm{He}$ [11], Bose-Einstein condensates [12-16], Josephson tunnel junctions [17-22], superconducting films [23] and ferroelectric materials [24] among others. This framework has also been proven to be adequate to shed light on supercritical and subcritical out-of-equilibrium bifurcations performed in optical Kerr-like systems [25] and in thermo-convective systems [26-31]. In general, the $\mathrm{KZ}$ mechanism [2,3] might be suitable to predict a scaling of the correlation length after a quenched bifurcation. This correlation length is related to the size of the 'domains' of a degenerate broken symmetry phase, which is of fundamental and applied interest in many fields of science and technology. On the basis of this mechanism, as the threshold of the bifurcation is crossed, topological defects arise throughout the space-time and become frozen

4 Author to whom any correspondence should be addressed. due to causal limitations. Topological defects [32-34] are ubiquitous in symmetry breaking phase transitions and can be understood as intrinsic non-linearities which in the present study correspond to phase singularities (the integral of the phase gradient along a closed path which encircles the defect is non-zero). According to Joets and Ribotta $[35,36]$ defects are stable localized states coming from the more symmetric state that are able to survive the critical point as relics with a reduced symmetry.

Subcritical bifurcations exhibit a wide variety of coherent structures that may coexist with the stable pattern. These coherent phenomena include fronts (1D and 2D) and localized patterns (pulses, solitons and waves) that may appear at the core of such instabilities. Likewise, this subcritical behavior has been observed experimentally or numerically in a wide range of physical systems such as fluids [37-41], granular materials [42], optical systems [43, 44], ferromagnetic materials [45, 46] and mechanical systems [47]. On the other hand, the quintic Ginzburg-Landau equation has succeeded in describing multi-stability in many dynamical systems [31, 48-57]. In general, subcritical bifurcations appear naturally in models with non-local interactions which, in turn, arise from the reduction of degrees of freedom in complex systems [31]. 
The cosmological implications of a subcritical scenario inspire deep respect if we move backwards to the first instants after the Big Bang, where the nature of such instability would be able to generate the present morphology of the Universe. Subcriticality at the beginning of time has been tackled by Rajantie [58] and Vachaspati [59]. In addition, if an inflationary universe is contemplated through a bubble nucleation process, which is distinctive of a first-order spontaneous symmetry breaking bifurcation, a nearly exponential growth regime of the universe is expected [60-64]. Despite the fact that much controversy has arisen about the rivalry between inflation and topological defect creation processes $[65,66]$, coexistence between defect creation and inflation has been held by some cosmologists [67-70].

In the present study, a subcritical approach to the $\mathrm{KZ}$ mechanism from the annihilation process of topological defects and the correlation of the average amplitude is performed. It is found that the $\mathrm{KZ}$ freezing dynamics concurs with an inflation-like regime before the new state is achieved at a lower rate, which is set by the final control parameter value. Besides, before entering this fast exponential growth of the order parameter, the amplitude of the vacuum fluctuations follows adiabatically the stationary amplitude, which is non-null due to an additive noise to simulate the fluctuations themselves.

In a subcritical bifurcation, excited states become more favored with respect to the vacuum as the transition point is approached. However, it is assumed that far enough from this transition point the correlation length $\xi$ and the relaxation time $\tau$ should follow the standard second-order phase transition critical scalings:

$$
\xi \sim|\varepsilon|^{-v} ; \quad \tau \sim|\varepsilon|^{-z \nu} ; \quad \varepsilon<0 .
$$

In the mean-field theory, $z v=1$ and $v=1 / 2$, consequently the dynamical exponent [71] becomes $z=2$. Nevertheless, the critical exponent $v$ which determines the correlation length decay is expected to be reduced in a subcritical bifurcation according to previous reports [26, 29]. In the present study the subcritical critical parameters $v$ and $z$ will be determined and used to predict the quenched dynamics. How the causal effects coming from the existence of a limiting speed in the system affect the selection of a length scale after a quench has been extensively described from several points of view in previous reports $[2-4,28,58,72]$, hence it will be skipped here on purpose. As a matter of fact, the $\mathrm{KZ}$ theory predicts that the mean size of domains after the transition (also known as the frozen correlation length) scales with the quench rate as:

$$
\xi_{\mathrm{KZ}} \sim \mu^{-v /(1+z v)} .
$$

The interval of quench rates where this relation holds will be called the KZ-scaling regime.

In this paper, the $\mathrm{KZ}$ mechanism is tested when a multi-stable region of the control parameter $\varepsilon$ is crossed at a finite constant rate $\mu$. The initial $\varepsilon$ is taken below and close to this region, and corresponds to a bi-stable region. However, our initial condition is vacuum-like. The final $\varepsilon$ is taken above and close to the locus where the vacuum state becomes unstable. Our aim is to search for the consequences of the KZ mechanism in this complex system, as well as to characterize the coexisting inflationary-like dynamics. For this purpose, this work is performed in a one-dimensional (1D) quintic Ginzburg-Landau equation. It is observed here that the correlation 'freezes' at the time interval $\left[-t_{\mathrm{o}}^{\mathrm{i}}, t_{\mathrm{o}}^{\mathrm{f}}\right]$ in agreement with the predicted critical parameters obtained from the quasistatic study. It is found that, the departure from adiabaticity spans a slightly broader range. Above the inflationary-like stage, in the subsequent asymptotic regime, the system follows a free-relaxation dynamics according to the growth rate of the amplitude at the final control parameter value and the defect annihilation process. With regard to the present numerical results, it should be mentioned that while some experiments are similar to our model (e.g. [31]), others are interesting candidates [15] because they may present the same kind of phenomenology (e.g. pulses and/or solitons). Owing to the fact that there has been scarce evidence in determining experimentally the critical $\mathrm{KZ}$ freezing parameters from the nature of the fluctuations before the transition point is effectively crossed [29], more experimental work might be devoted to this matter.

According to our previous work in a large array of non-locally coupled convective oscillators [31] where we had modeled an experiment undergoing a secondary subcritical bifurcation, here we take again the 1D cubic-quintic Ginzburg-Landau equation [48] but in the continuum case:

$$
\begin{aligned}
\tau\left(\partial_{t} A\right)= & \varepsilon A+\left(b_{3}+\mathrm{i} c_{3}\right)|A|^{2} A-\left(b_{5}-\mathrm{i} c_{5}\right)|A|^{4} A \\
& +\left(b_{1}+\mathrm{i} c_{1}\right) \partial_{x x} A+\zeta .
\end{aligned}
$$

The unstable mode $A$ of the new pattern is represented by its phase $\phi$ and amplitude $\rho: A=\rho \mathrm{e}^{\mathrm{i} \phi}$. The control parameter is $\varepsilon$, which in the former study was related to the temperature difference. We implement a second-order Adams-Bashforth scheme with periodic boundary conditions for the phase and the amplitude equations. The space discretization is fixed at $\Delta x=1$ (for a total length $L=400$ ), meanwhile the time steps $\Delta t \leq 0.01$ are adaptive. Hereafter, we will omit the 'arbitrary units' labeling both in the text and in the figures. The coefficients in equation (3) are $\tau=1 ; c_{1}=0 ; c_{3}=1$; $c_{5}=1 ; b_{1}=1 ; b_{3}=3 ; b_{5}=2.75$, and $\zeta=\zeta(x, t)$ is a white Gaussian additive noise that is imposed independently on the real and imaginary components of $A$. This Gaussian noise obeys $\langle\zeta(x, t)\rangle=0$ and $\left\langle\zeta(x, t) \zeta\left(x^{\prime}, t^{\prime}\right)\right\rangle=\sqrt{\Lambda} \delta\left(x-x^{\prime}\right) \delta(t-$ $\left.t^{\prime}\right)$, where $\delta(t)$ is the Dirac distribution and $\Lambda$ is the amplitude of the noise which depends on the control parameter according to experimental and numerical studies [73]. For this particular set of parameters, the formation of coherent regions of the new state $(\rho \neq 0)$ is favored by the noise. Below the threshold in quasistatic regimes these new coherent regions are grown from the vacuum state $(\rho=0)$ through its fluctuations (noise). Hence, the fluctuations in our system might be equivalent to the temperature fluctuations in equilibrium condensed matter systems. Still, it is important to emphasize that these thermal fluctuations are usually much smaller than others appearing in pattern-forming (out-of-equilibrium) systems [48], and also 

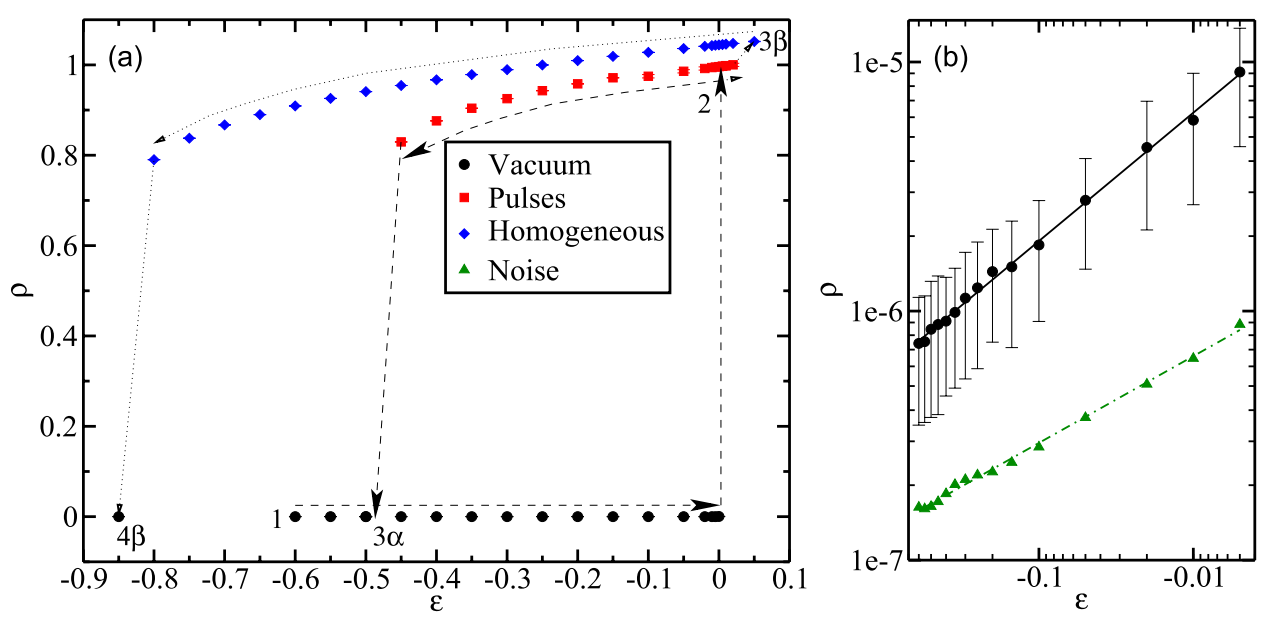

Figure 1. (a) Hysteresis cycle for the order parameter corresponding to the interval of the control parameters concerned in the present work. The initial state (1) is a vacuum one with a very small and constant amplitude imposed by noise (fluctuations). The homogeneous state corresponds to a high and constant amplitude pattern, and the pulse regime corresponds to the presence of localized patterns coexisting with the vacuum state. The two hysteresis cycles are described by the sequences: $1 \rightarrow 2 \rightarrow 3 \alpha$ (dashed line) and $1 \rightarrow 2 \rightarrow 3 \beta \rightarrow 4 \beta$ (dashed line up to (2) and dotted line). (b) The order parameter (circles) in the vacuum state. The solid line is a fit to a power law $\propto(-\varepsilon)^{-0.52 \pm 0.01}$. A plot for the noise amplitude (triangles) is provided for comparison along with a fit (dotted-dashed line) to a power law $\propto(-\varepsilon)^{-0.35 \pm 0.01}$.

that they are not taking part in the process of defect generation in the framework of the Ginzburg-Landau theory [74].

On the whole, we choose these specific parameters because on the one hand, in our quenches, the ending control parameter value corresponds to a bi-stable region, where pulses might emerge and on the other hand they provide a very rich phenomenology for this kind of model, which can represent several experimental situations.

\section{From the quasistatic characterization to the Kibble-Zurek mechanism}

In the region of interest of this work $\varepsilon \in[-0.85,0.02]$, we can attain at least three well-defined states, which can be connected by quasistatic changes of the control parameter $\varepsilon$ (figure 1(a)): (i) the vacuum state which, provided that there were no fluctuations, would be identically null $(\rho=0)$; (ii) the homogeneous state which has a non-zero constant amplitude with a periodically varying phase; (iii) the pulse state which consists of a number (between 18 and 22) of localized pulses. In the former two states the order parameter is the average amplitude and in the latter it is the average value of the pulse maxima.

Figure 1(a) shows two hysteresis cycles built from the sequences: $1 \rightarrow 2 \rightarrow 3 \alpha$ (dashed line) and $1 \rightarrow 2 \rightarrow 3 \beta \rightarrow$ $4 \beta$ (dashed line up to (2) and onwards dotted line). The quasistatic characterization starts with a zero initial condition at $\varepsilon=-0.6$. After the permanent state is reached, it will be used as the initial state for the next point of the hysteresis cycle. Thus, the control parameter is increased (decreased) slowly to reach quasistatically the permanent states. In this way, we follow two different sequences $(\alpha, \beta$; see figure 1) to characterize the bifurcation diagram. In the following section we are going to perform quenches from a vacuum state at $\varepsilon=-0.5$, which is already in a bi-stable region, to a final

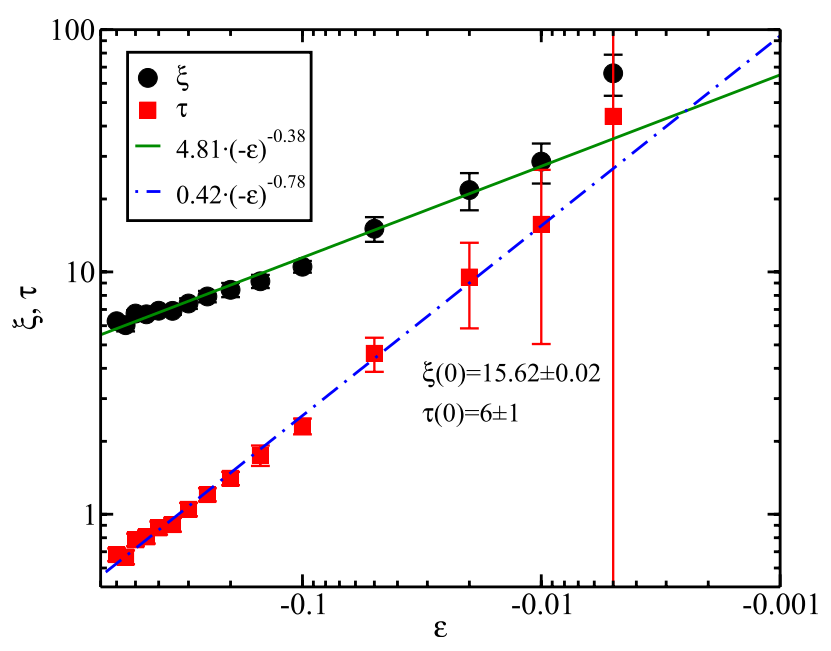

Figure 2. In the vacuum state, the correlation lengths $\xi$ (circles) and relaxation times $\tau$ (squares) are obtained at the quasistatic regimes. The solid line is the fit to a power law $\propto(-\varepsilon)^{-0.38 \pm 0.01}$, and the dotted-dashed line is the fit to a power law $\propto(-\varepsilon)^{-0.78 \pm 0.01}$. The corresponding values at the threshold $\varepsilon=0$ are given.

control parameter value $(\varepsilon=0.01)$ where the tri-stability is lost due to a destabilization of the vacuum state.

For the purpose of determining the critical exponents $z$ and $v$ of equation (1), the states obtained for each control parameter $\varepsilon$ in the previous hysteresis cycle are prepared as the initial state in the following way: we use the same phase as the permanent state at $\varepsilon$ but with an amplitude 20 times bigger. This kind of initial state will from now be called an amplified noisy state. We let this initial condition evolve until the average amplitude $\langle\rho\rangle_{x}$ decays to $1 / e$ of the initial one. This lapse of time is the relaxation time $\tau$ (figure 2, squares).

Next, we measure the stationary correlation length. For this reason, we measure the relaxation times for sinusoidal initial conditions with all the possible wavenumbers (data not 


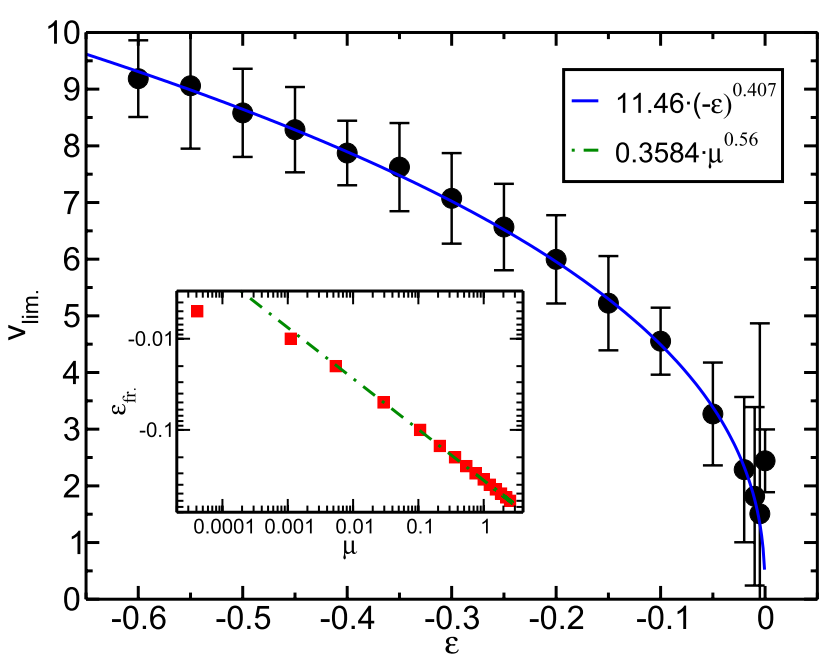

Figure 3. Limit speed $v_{\text {lim }}$ of the system for perturbations of the vacuum state versus the control parameter $\varepsilon$. The solid line is a fit to a power law $\propto(-\varepsilon)^{0.407 \pm 0.004}$. The inset shows the values of the freezing control parameter $\varepsilon_{\mathrm{fr}}$. versus the crossing rate $\mu$. The dotted-dashed line is the result given by $\varepsilon_{\mathrm{fr} .}=-\left(\nu \xi_{\mathrm{o}} \mu / v_{\mathrm{o}}\right)^{1 /(1+z v)}=-0.36 \mu^{0.56}$.

shown). Then, we set the initial state as an amplified noisy state with an amplitude 40 times bigger than the permanent state attained at $\varepsilon$. Again, we let it evolve for a long enough time, which is defined by the maximum time necessary to erase the initial conditions (which were obtained from the sinusoidal initial conditions simulations), and we measure the correlation length $\xi$ (figure 2, circles).

In figure 2, we observe that, for values bigger than -0.01 , the behavior deviates slightly from the classical power law for second-order phase transitions. This is due to the subcriticality, which actually makes finite the values of the correlation length and the relaxation time at $\varepsilon=0$. Note that the error bars for small $|\varepsilon|$ are much bigger than for $\varepsilon=0$. From the fits to the data in the power-law region (i.e. $\varepsilon \leq$ -0.01 ), we obtain according to equation (1), $v=0.38 \pm 0.01$ and $z=2.05 \pm 0.06$. These findings are consistent with the effect that the subcriticality may have on the critical exponent $v$, as suggested by previous experiments [26, 29]. Meanwhile, the dynamical exponent $z$ is, within the statistical error, the one predicted by mean-field theory.

In our system, the limiting speed is not the speed of light in vacuum. Instead, it is the speed of propagation of the perturbations of the order parameter in the vacuum state $v_{\text {lim }}=\frac{\xi}{\tau}$ [3]. In figure 3, we show these values as well as the fit in the power-law region that gives $v_{\mathrm{o}}(-\varepsilon)^{0.407 \pm 0.004}$, where the exponent provides a better resolution for $(z-1) v$ and $v_{\mathrm{o}}=\exp (2.44 \pm 0.01)$. It must be noted that the region close to the threshold $\varepsilon=0$ does not follow the power law due to the subcritical character of the bifurcation.

In a supercritical bifurcation, or in a second-order phase transition, the limiting speed goes to zero as $\varepsilon \rightarrow 0$. This means that even at very low crossing rates, the fluctuations in the vacuum cannot synchronize during the transition. The regime at which the dynamics is slowed down starts at $\varepsilon=$ $\varepsilon_{\mathrm{fr} .}$, which is determined from the $\mathrm{KZ}$ mechanism as $\varepsilon_{\mathrm{fr} .}=$

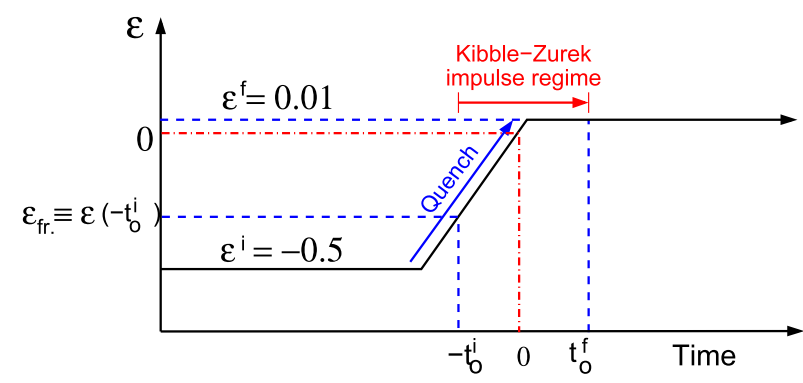

Figure 4. Sketch of the quenching process, where $\varepsilon=0$ is the quasistationary threshold and $\varepsilon^{\mathrm{i}, \mathrm{f}}$ are the initial and final values of the control parameter at a crossing rate $\mu=\frac{\mathrm{d} \varepsilon}{\mathrm{d} t}$. The freezing dynamics takes place in the interval $\left[-t_{\mathrm{o}}^{\mathrm{i}}, t_{\mathrm{o}}^{\mathrm{f}}\right]$.

$-\left(\frac{\nu \xi_{\mathrm{o}} \mu}{v_{\mathrm{o}}}\right)^{1 /(1+z v)}$ [3]. In figure 3 (inset), the values for $\varepsilon_{\mathrm{fr}}$ coming directly from the data are shown (squares) together with the values from the predicted model (dotted-dashed line). From this inset, we will consider in section 3 , where a linear ramp of the control parameter is imposed, that: (i) if the initial control parameter value for the quench is $\varepsilon=$ -0.5 , then the maximum quench rate $\mu$ is around 1.8 ; for higher crossing rates, the $\mathrm{KZ}$ freezing regime starts from the very beginning of the quench and consequently the frozen correlation length should be constant; and (ii) the minimum limit speed occurs at around $\varepsilon=-0.005$, which corresponds to a quench rate of approximately $3 \times 10^{-5}$. As a consequence, crossing rates smaller than this value will not be able to freeze the correlation length and the system will follow adiabatically the quasistatic results.

\section{Quenched dynamics: the Kibble-Zurek mechanism, defect annihilation and inflationary dynamics}

According to the aforementioned argument, here the control parameter becomes time dependent. In the framework represented in figure 1, quenches are performed with a linear dependence of the control parameter on the crossing rate $\varepsilon(t)=\mu t$ along the transition through the threshold $\varepsilon=0$ (see sketch in figure 4) from $\varepsilon^{i}=-0.5$ to $\varepsilon^{f}=0.01$. To ensure valid equivalent initial states (conditions) [75], we prepare an amplified noisy state and we let it evolve freely, before imposing the quench, over six units of time, which is longer than the maximum relaxation time at $\varepsilon^{\mathrm{i}}$. This free evolution period sets a practical limit to the observation of the KZ-scaling regime at relatively high $\mu$ (in our case $\mu_{\text {max. }}=$ 0.12 ). When the quench is finished at $\varepsilon^{\mathrm{f}}$, the system is allowed to stay until the end of the simulation run.

In figure 5, representative spatio-temporal diagrams of $\ln (\rho)$ at two quench rates $(0.001$ and 0.02$)$ are shown. Hereafter, all the simulations and quantitative analyses are performed over ten realizations for each $\mu$, as a compromise between the required computational times and the statistics. The results shown correspond to the interval $\mu=[0.001,0.1]$, where, under these numerical conditions, the KZ-scaling is expected. Coherent structures appear close to the threshold (see growing brighter regions in figure 5(a)). Dark regions in 

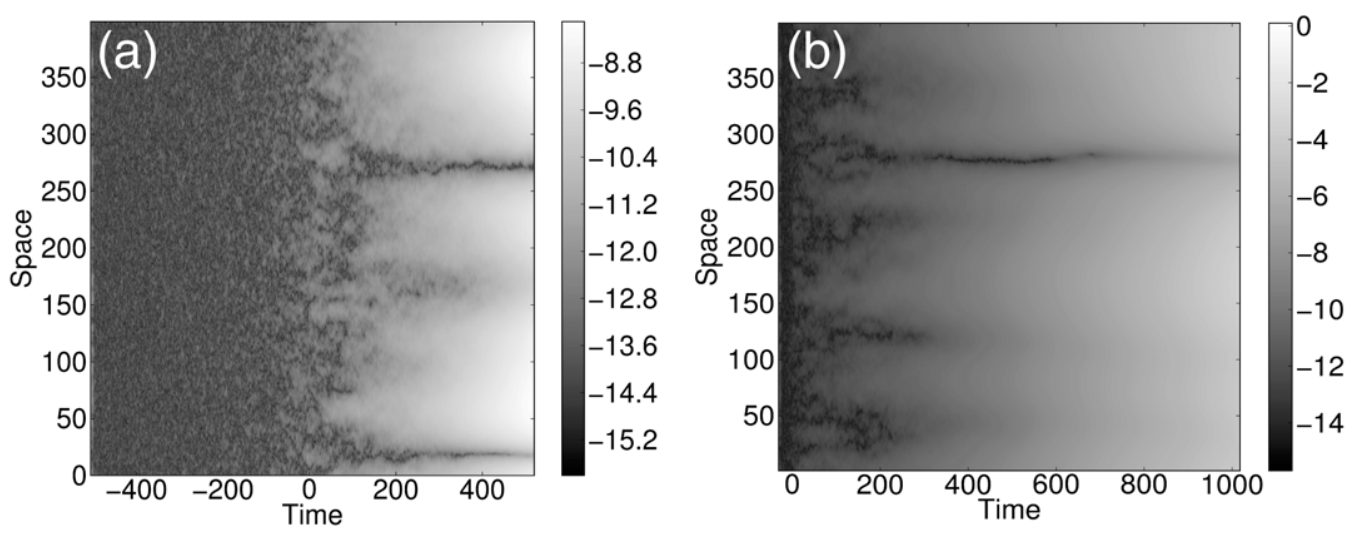

Figure 5. Spatio-temporal diagrams of the logarithm of the amplitude at two crossing rates: (a) $\mu=0.001$, (b) $\mu=0.02$. In both cases, the quench starts after six units of time from the beginning of the spatio-temporal diagram.

figure 5 correspond to fluctuations of a very low amplitude in the vacuum-like state. However, once the system crosses $\varepsilon=0$, relics of the vacuum state remain as topological defects in the new growing state (see the elongated darker structures). It is observed that, for sufficiently long simulation runs, as the crossing rate is increased the system bifurcates directly towards the homogeneous state (data not shown) of the bifurcation diagram (figure 1), whereas for crossing rates $\mu>0.02$, the first stage of noise and defects is similarly short.

In the following, we show that the number of topological defects $(N)$ that remain along the freezing regime depends on the crossing rate $\left(\mu=\frac{\mathrm{d} \varepsilon}{\mathrm{d} t}\right)$. The initial time of this freezing regime, also called the $\mathrm{KZ}$ impulse regime, according to the theoretical prediction $[2,3]$ is $-t_{\mathrm{o}}^{\mathrm{i}}$ and can be obtained from the fact that $\varepsilon_{\mathrm{fr}}=-t_{\mathrm{o}}^{\mathrm{i}} \mu$. Thus, according to the expression of the freezing control parameter $\varepsilon_{\mathrm{fr}}$. found in section $2, t_{\mathrm{o}}^{\mathrm{i}}=$ $0.36 \mu^{-0.44}$. From now on, results on the quenched dynamics close to the threshold will be time scaled with $t_{\mathrm{o}}^{\mathrm{i}}$.

The out-of-equilibrium dynamics is characterized from: (i) the order parameter, which is the average amplitude $\langle\rho\rangle_{x}$ (the average is performed along the space coordinate $x$ and for the different realizations) and which provides evidence of an inflationary-like behavior that is triggered when the adiabatic regime is finished, (ii) the number of topological defects, $N(t)$, which are measured straightforwardly from the null values of the real and imaginary parts of $A(x, t)$ by analyzing the change in sign of its spatial derivative and, finally, (iii) the averaged two-point correlation function $\langle\rho(x, t) \rho(x+\lambda, t)\rangle_{x}$ (where $\lambda$ is the spatial lag) along time which will allow us to determine the correlation length at the threshold $\xi_{\rho}(0)$ [15].

From the analysis of the average amplitude $\langle\rho\rangle_{x}$ (figure 6), during the stage before the transition point is crossed, it can be observed that different quenches lead to the same dynamics for initial times (see dashed line in figure 6). This power-law dynamics corresponds to an adiabatic behavior where the amplitude depends on $\varepsilon=\mu t$, but not on $\mu$. After some time, the adiabatic behavior is lost and the amplitudes start to change their growth as a function of $\mu$. This occurs before the beginning of the causally induced $\mathrm{KZ}$ impulse regime $-t_{0}^{\mathrm{i}}$ (crosses in figure 6), which follow the same scaling (see the dotted-dashed line in figure 6). If

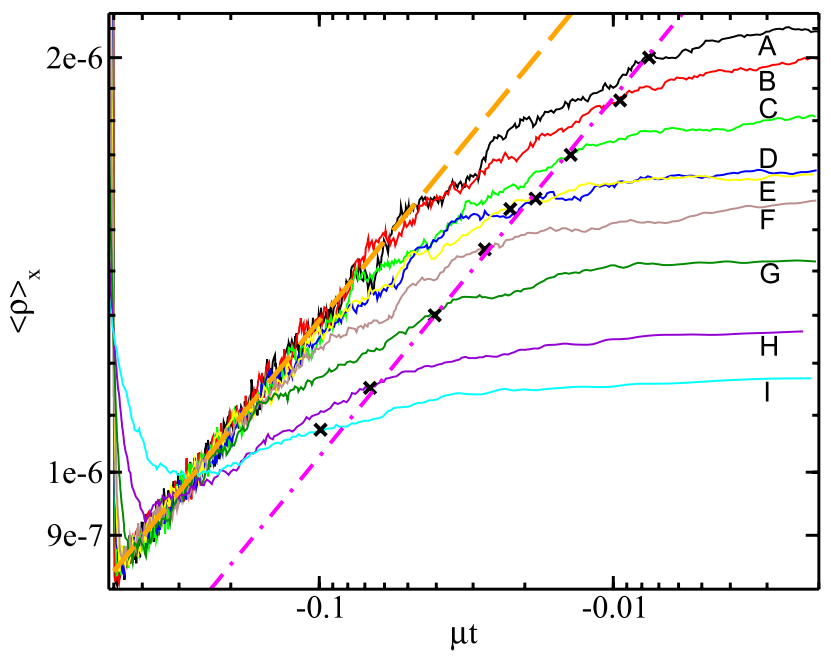

Figure 6. Average amplitude $\langle\rho\rangle_{x}$ versus $\mu t$ for several crossing rates in the KZ-scaling regime. Data curves are labeled according to $\mu$ (A: 0.001, B: 0.0015, C: 0.003, D: 0.005, E: 0.007, F: 0.01, G: 0.02, H: 0.05 and I: 0.1 ). The dashed line is a fit to a power law $7.1 \times 10^{-7}(-\mu t)^{-0.26 \pm 0.02}$, the crosses $(\mathbf{x})$ indicate the beginning of the expected KZ freezing regime at $t=t_{\mathrm{o}}^{\mathrm{i}}=0.36 \mu^{-0.44}$, and the dotted-dashed line is a power-law fit using the same exponent as for the dashed line. All quenches start at $\mu t=-0.5$.

we take into account the uncertainty of the data, the adiabatic regime is measured to end at $(-2.1 \pm 0.1) t_{\mathrm{o}}^{\mathrm{i}}$.

In figure 7 , we represent the normalized average amplitude as a function of the dimensionless time $t / t_{0}^{\mathrm{i}}$ at several quench rates. In this case, the data before the transition point is crossed collapse onto a single curve. Another relevant result is that we are able to identify an inflationary-like dynamics in the vicinity of the threshold $(t=0)$ where the normalized average amplitude converges to a faster exponential growth: $\frac{\langle\rho\rangle_{x}}{\langle\rho\rangle_{x}(0)}=\exp \left(0.08 t / t_{\mathrm{o}}^{\mathrm{i}}\right)$ (see the solid straight line in figure $7(b)$ ). The inflation of the amplitude occurs within a symmetric interval with regard to $t=0$. Furthermore, from figure 7(b) we observe that the inflation-like dynamics regime approximately spans from $-2 t_{\mathrm{o}}^{\mathrm{i}}$ to $2 t_{\mathrm{o}}^{\mathrm{i}}$, in close agreement with both the end of the adiabatic regime and the beginning of the free-relaxation regime, respectively. 

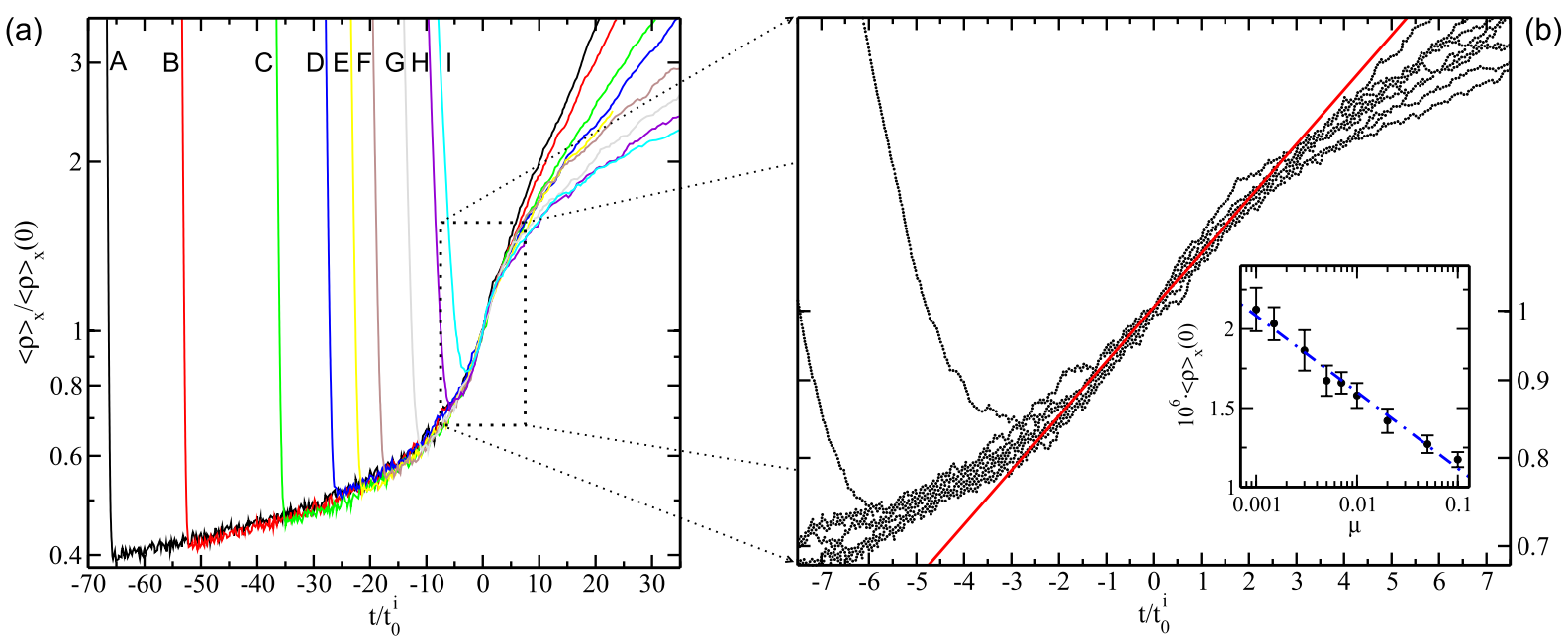

Figure 7. (a) Normalized average amplitude $\frac{\langle\rho\rangle_{x}}{\langle\rho\rangle_{x}(0)}$ versus dimensionless time (with $t_{\mathrm{o}}^{\mathrm{i}}=0.36 \mu^{-0.44}$ ). Before the transition, all data collapse. The coding (A-I) for $\mu$ follows that of figure 6. (b) Zoom of a region which encloses the inflationary-like regime fitted to $\exp \left(0.08 t / t_{\mathrm{i}}^{\mathrm{i}}\right)$ (solid line). The inset shows the average amplitude at the transition point $(t=0)$. The dashed line corresponds to $6.36 \times 10^{-7}[1-0.33 \ln \mu]$.

Afterward, when this inflationary-like dynamics comes to an end, the average amplitude follows the growth rate for small amplitudes $\left(\langle\rho\rangle_{x} \sim \exp (0.008 t)\right)$ which corresponds to the final control parameter $\varepsilon^{\mathrm{f}}=0.01$ until non-linear effects come into play, when a transition takes place towards the branch of the homogeneous state. With regard to this fact, it is important to emphasize that pulses are not recovered in the quenched transitions in our range of crossing quench rates, not even for the slowest ones.

From the normalized number of defects $N / N(0)$, three regimes can be distinguished in figure 8. Firstly, prior to the inflationary-like dynamics regime characterized from the analysis of figure 7, the rate of defect annihilation is given by a power law which corresponds to the stage where the system is still able to follow adiabatically the constraints imposed by the quench (see the dashed line in figure 8). Secondly, from around $-2 t_{\mathrm{o}}^{\mathrm{i}}$ the defect annihilation rate is speeded up. The inset in figure 8 shows the power-law fits of $N(t)$ in front of $\mu$ measured at the boundaries that define the KZ freezing regime $\left(-t_{\mathrm{o}}^{\mathrm{i}}\right.$ and $\left.t_{\mathrm{o}}^{\mathrm{f}} \approx t_{\mathrm{o}}^{\mathrm{i}}\right)$ and at the threshold $(t=$ $0)$. These fits give the same exponents within the statistical error, thus the density of defects is a proper measure to test the KZ mechanism as will be shown below. Thirdly, when the quenched dynamics has finished, the defect annihilation process follows a free-relaxation regime with an exponential decay in accordance with a previous report [76].

At the threshold $(t=0)$ of the quenched transition, where the freezing dynamics for the perturbation propagation of the order parameter coexists with its fast inflation-like local growth, we can determine the number of defects $N(0)$, which has to be inversely proportional to the $d$-power of the correlation length, where $d$ is the geometrical dimension of the system. In $1 \mathrm{D}$, the correlation length is given by $\xi(0)=$ $L / N(0)$ ( $L$ is the size of the system). In figure 9 , we show that the $\mathrm{KZ}$ mechanism is accomplished for quenches within the range of $\mu$ where the KZ-scaling can be tested. Moreover, the power law $\xi(0) \equiv L / N(0) \sim \mu^{-0.22 \pm 0.02}$ agrees with the

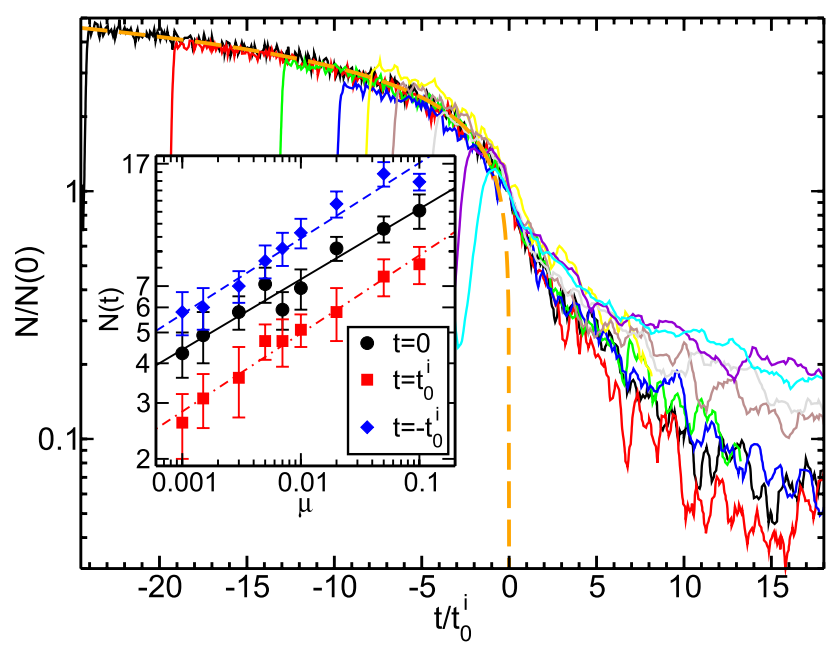

Figure 8. Normalized number of defects $N / N(0)$ versus $t / t_{0}^{\mathrm{i}}$. The dashed line accounts for the adiabatic dynamics prior to the $\mathrm{KZ}$ impulse regime fitted to a power law $\left(-t / t_{\mathrm{o}}^{\mathrm{i}}\right)^{0.4}$. The inset plots $N(t)$ versus $\mu$ for $t=-t_{\mathrm{o}}^{\mathrm{i}}, 0, t_{\mathrm{o}}^{\mathrm{i}}$ and their fits to power laws.

critical exponents given by the quasistatic study $(-0.213 \pm$ 0.002 ) and by equation (2). It should be emphasized that a similar fit for $\xi$ is recovered at the boundaries of the $\mathrm{KZ}$ impulse regime $\left[-t_{\mathrm{o}}^{\mathrm{i}}, t_{\mathrm{o}}^{\mathrm{f}}\right]$ (where $t_{\mathrm{o}}^{\mathrm{f}} \approx t_{\mathrm{o}}^{\mathrm{i}}=0.36 \mu^{-0.44}$ ) within statistical error (see also the inset in figure 8). Out of this boundary, the KZ mechanism is accomplished with a larger error (e.g. at $2 t_{\mathrm{o}}^{\mathrm{i}}, L / N(0) \sim \mu^{-0.28 \pm 0.04}$ ). Furthermore, the correlation length given by the averaged two-point correlation function [15] of the amplitude at $t=0$ allows us to check again the $\mathrm{KZ}$ mechanism $\xi_{\rho}(0) \sim \mu^{-0.21 \pm 0.01}$ (see figure 9).

\section{Concluding remarks}

The Kibble-Zurek mechanism has been studied numerically from the point of view of quenched transitions 


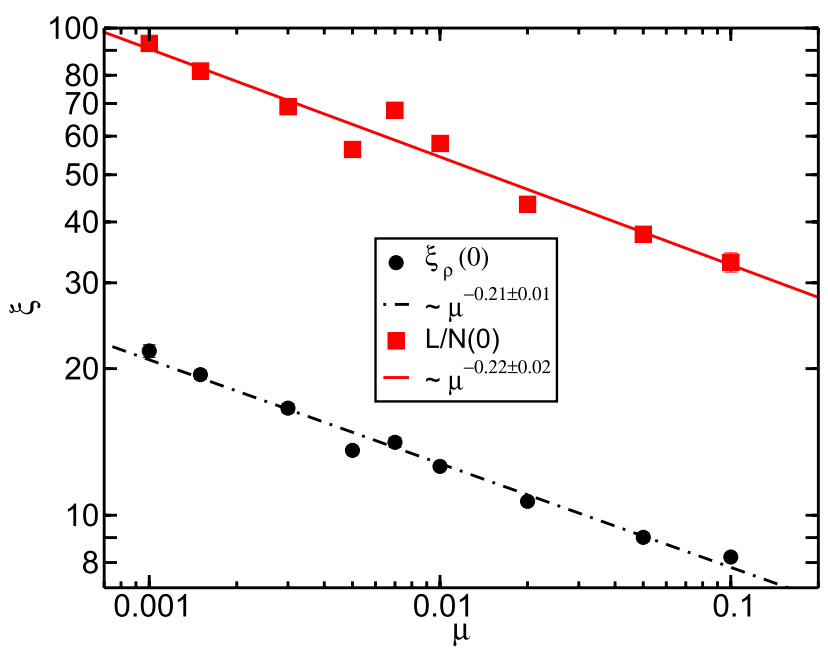

Figure 9. Correlation length $\xi$ versus the crossing rate $\mu$. At the threshold $(t=0), \xi_{\rho}(0)$ is obtained from the averaged two-point correlation function of the amplitude, and $L / N(0)$ is obtained from the number of defects $N(0)$ in a $1 \mathrm{D}$ system of size $L$. The solid line corresponds to a power-law fit $\propto \mu^{-0.22 \pm 0.02}$, and the dotted-dashed line corresponds to a power-law fit $\propto \mu^{-0.21 \pm 0.01}$.

through a subcritical bifurcation in a 1D quintic complex Ginzburg-Landau equation. This equation has successfully described reactive-diffusive systems in continuum and discrete systems where the coexistence of different regimes is possible. Under the quenched transitions, the vacuum state becomes unstable towards a homogeneous pattern throughout the formation of topological defects. The critical exponents of the $\mathrm{KZ}$ mechanism and the boundaries of the KZ-scaling regime determined from the quenched dynamics are in consonance with the predicted values given by the former quasistatic study performed before the transition point. On the one hand, the quasistatic regimes allow us to determine the characteristic magnitudes ( $\xi$ and $\tau$ ) that yield the critical exponents in the classical theory of phase transitions $(v=$ $0.38 \pm 0.02$ and $z=2.05 \pm 0.06$ ), and hence according to the $\mathrm{KZ}$ mechanism we obtain $\xi \sim \mu^{-0.21}$, which is in perfect agreement with the numerical results obtained from the quenched study (figure 9). On the other hand, they provide the horizon (the limiting speed of the system is given by the 'sound speed' $v_{\lim }=\xi / \tau$ ) for each $\varepsilon$ in order to consider the role of causal effects during quenches at the freezing time scale given by $t_{\mathrm{o}}^{\mathrm{i}}=0.36 \mu^{-0.44}$.

We found that the dynamics of the order parameter, across this subcritical threshold, follows three well-defined regimes (figure 10), namely the adiabatic regime, the inflationary-like regime (which encloses the $\mathrm{KZ}$ impulse regime) and the free-relaxation regime. The inflation-like dynamics is verified from a sharp nearly exponential growth of the average amplitude close to the threshold in between two slower regimes. This faster growth could be triggered by the loss of adiabaticity when the limit speed becomes comparable to the speed of the growing horizon. Besides, it should be emphasized that the de-correlation of different regions of the system makes its interactions more local and consequently the effective subcriticality could become smaller [31]. Thus, the

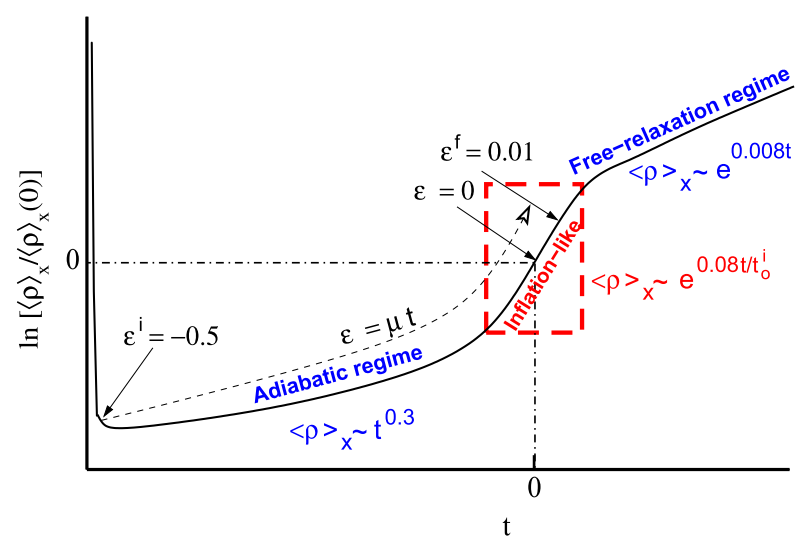

Figure 10. Sketch of the order parameter increase $\frac{\langle\rho\rangle_{x}}{\langle\rho\rangle_{x}(0)}$ in the logarithmic scale versus time for a typical value of the crossing rate. The dashed arrow represents the increasing control parameter $\varepsilon$ throughout the quench. Three regimes are distinguished and their corresponding fittings: the adiabatic regime, the inflation-like dynamics centered at the critical point $(t=0)$ and the free-relaxation regime well beyond the end of the quench.

super-cooled state becomes unstable and the fast exponential growth begins. In terms of the cosmological e-folds as a suitable measure of inflation [64, 77, 78], from our simulations we find that, before the end of the inflation-like regime, the number of e-folds for the order parameter is small $(\approx 0.3)$, while the number of defects scales down by a factor of $(\approx 4.3)$ independently of the quench rate value. Probably, the number of e-folds is related to the subcriticality of the model which could be increased by varying its parameters.

To summarize, we have shown that the quenching dynamics is in agreement with both the $\mathrm{KZ}$ mechanism from the number of frozen defects $N(0)$ measured at the threshold which yields $\xi(0) \equiv L / N(0) \sim \mu^{-0.22 \pm 0.02}$ and from the averaged two-point correlation function of the amplitude $\left(\xi_{\rho}(0) \sim \mu^{-0.21 \pm 0.01}\right)$. Moreover, it has been tested that the $\mathrm{KZ}$ impulse regime occurs from $-t_{\mathrm{o}}^{\mathrm{i}}$ to $t_{\mathrm{o}}^{\mathrm{f}} \approx t_{\mathrm{o}}^{\mathrm{i}}$, nonetheless, according to the average amplitude, we show that the inflationary-like dynamics is confined to a wider region $\left[-2.1 t_{\mathrm{o}}^{\mathrm{i}}, 2.1 t_{\mathrm{o}}^{\mathrm{i}}\right]$ that starts at the end of the adiabatic regime until the free-relaxation regime begins. With regard to the cosmological analogy, an inflationary scenario can be attained from an unstable vacuum state [79], or from a super-cooled vacuum state [61,63], meanwhile in our system the dynamics is externally triggered from a stable vacuum state. Indeed, we consider that the degeneracy at the critical point in this subcritical framework accounts for our inflation-like dynamics, which is a compelling physical response to the $\mathrm{KZ}$ mechanism in quenched processes and calls for further research.

\section{Acknowledgments}

MAM and WGV contributed equally to this work. The authors acknowledge partial financial support from the Spanish Government (grant no. FIS2011-24642), FONDECYT 1120764, Millennium Scientific Initiative, P10-061-F, Basal Program 
Center for Development of Nanoscience and Nanotechnology (CEDENNA) and UTA-project 8750-12.

\section{References}

[1] Kibble T W B 1976 Topology of cosmic domains and strings J. Phys. A: Math. Gen. 9 1387-98

[2] Zurek W H 1985 Cosmological experiments in superfluid helium? Nature 317 505-8

[3] Zurek W H 1996 Cosmological experiments in condensed matter systems Phys. Rep. 276 177-221

[4] Kibble T W B 2007 Phase-transition dynamics in the lab and the universe Phys. Today 6047

[5] Chuang I, Dürrer R, Turok N and Yurke B 1991 Cosmology in the laboratory: defect dynamics in liquid crystals Science 251 1336-42

[6] Bowick M J, Chandar L, Schiff E A and Srivastava A M 1994 The cosmological Kibble mechanism in the laboratory: string formation in liquid crystals Science 263 943-5

[7] Chen X, Hamlington B D and Shen A Q 2008 Isotropic-to-nematic phase transition in a liquid-crystal droplet Langmuir 24 541-6

[8] Eltsov V B, Kibble T W B, Krusius M, Ruutu V M H and Volovik G E 2000 Composite defect extends analogy between cosmology and He-3 Phys. Rev. Lett. 85 4739-42

[9] Eltsov V B, Krusius M and Volovik G E 2005 Vortex formation and dynamics in superfluid ${ }^{3} \mathrm{He}$ and analogies in quantum field theory Prog. Low Temp. Phys. 15 1-137

[10] Eltsov V B, Hänninen R and Krusius M 2010 Vortices and other topological defects in non-equilibrium phase transitions of superfluid ${ }^{3} \mathrm{He}$ Physica C 470 803-8

[11] Hendry P C, Lawson N S, Lee R A M, McClintock P V E and Williams C D H 1994 Generation of defects in superfluid ${ }^{4} \mathrm{He}$ as an analogue of the formation of cosmic strings Nature 368 315-7

[12] Saito H, Kawaguchi Y and Ueda M 2007 Kibble-Zurek mechanism in a quenched ferromagnetic Bose-Einstein condensate Phys. Rev. A 76043613

[13] Weiler C N, Neely T W, Scherer D R, Bradley A S, Davis M J and Anderson B P 2008 Spontaneous vortices in the formation of Bose-Einstein condensates Nature 455 948-51

[14] Zurek W H 2009 Causality in condensates: gray solitons as relics of BEC formation Phys. Rev. Lett. 102105702

[15] Damski B and Zurek W H 2010 Soliton creation during a Bose-Einstein condensation Phys. Rev. Lett. 104160404

[16] Das A, Sabbatini J and Zurek W H 2012 Winding up superfluid in a torus via Bose Einstein condensation Sci. Rep. 2352

[17] Carmi R and Polturak E 1999 Search for spontaneous nucleation of magnetic flux during rapid cooling of $\mathrm{YBa}_{2} \mathrm{Cu}_{3} \mathrm{O}_{7}$ - delta films through $T_{\mathrm{c}}$ Phys. Rev. B 60 7595-600

[18] Monaco R, Mygind J and Rivers R J 2002 Zurek-Kibble domain structures: the dynamics of spontaneous vortex formation in annular Josephson tunnel junctions Phys. Rev. Lett. 89080603

[19] Monaco R, Mygind J and Rivers R J 2003 Spontaneous fluxon formation in annular Josephson tunnel junctions Phys. Rev. B 67104506

[20] Monaco R, Olsen U L, Mygind J, Rivers R J and Koshelets V P 2006 Saturation in the Zurek-Kibble mechanism for the spontaneous vortex formation in annular Josephson tunnel junctions Phys. Rev. Lett. 96180604

[21] Monaco R, Aaroe M, Mygind J, Rivers R J and Koshelets V P 2008 Spontaneous fluxon production in annular josephson tunnel junctions in the presence of a magnetic field Phys. Rev. B 77054509
[22] Monaco R, Mygind J, Rivers R J and Koshelets V P 2009 Spontaneous fluxoid formation in superconducting loops Phys. Rev. B 80180501

[23] Maniv A, Polturak E and Koren G 2003 Observation of magnetic flux generated spontaneusly during a rapid quench of superconducting films Phys. Rev. Lett. 91197001

[24] Griffin S M, Lilienblum M, Delaney K T, Kumagai Y, Fiebig M and Spaldin N A 2012 Scaling behavior and beyond equilibrium in the hexagonal manganites Phys. Rev. X 2041022

[25] Ducci S, Ramazza P L, González-Viñas W and Arecchi F T 1999 Order parameter fragmentation after a symmetry-breaking transition Phys. Rev. Lett. 83 5210-3

[26] Casado S, González-Viñas W, Mancini H and Boccaletti S 2001 Topological defects after a quench in a Bénard-Marangoni convection system Phys. Rev. E 63057301

[27] Casado S, González-Viñas W and Mancini H 2006 Testing the Kibble-Zurek mechanism in Rayleigh-Bénard convection Phys. Rev. E 74047101

[28] Casado S, González-Viñas W, Boccaletti S, Ramazza P L and Mancini H 2007 The birth of defects in pattern formation: testing of the Kibble-Zurek mechanism Eur. Phys. J. Spec. Top. 146 87-98

[29] González-Viñas W, Casado S, Burguete J, Mancini H and Boccaletti S 2001 Defect dynamics during a quench in a Bénard-Marangoni convection system Int. J. Bifurcation. Chaos 11 2887-94

[30] Miranda M A, Burguete J, González-Viñas W and Mancini H 2012 Exploring the Kibble-Zurek mechanism in a secondary bifurcation Int. J. Bifurcation Chaos 221250165

[31] Miranda M A, Burguete J, Mancini H and González-Viñas W 2013 Frozen dynamics and synchronization through a secondary symmetry-breaking bifurcation Phys. Rev. E 87032902

[32] Kléman M 1977 Points, Lignes, Parois (Orsay, France: Éditions de Physique)

[33] Mermin N D 1979 The topological theory of defects in ordered media Rev. Mod. Phys. 51 591-648

[34] Michel L 1980 Symmetry defects and broken symmetry. configurations. hidden symmetry Rev. Mod. Phys. 52 617-51

[35] Joets A and Ribotta R 1991 Localized bifurcations and defect instabilities in the convection of a nematic liquid crystal J. Stat. Phys. 64 981-1005

[36] Ribotta R, Belaidi A and Joets A 2004 Singularities, defects and chaos in organized fluids Geometry and Topology of Caustics-Caustics'02 ed S Janeczko and D Wiersma (Warsaw: Polish Academy of Sciences) pp 223-38

[37] Chen P and Wu K-A 2000 Subcritical bifurcations and nonlinear balloons in Faraday waves Phys. Rev. Lett. $853813-6$

[38] Boedo S 1999 Experimental verification of subcritical whirl bifurcation of a rotor supported on a fluid film bearing J. Tribol. 121 421-2

[39] Miranda M A and Burguete J 2008 Subcritical instabilities in a convective fluid layer under a quasi-one-dimensional heating Phys. Rev. E 78046305

[40] Miranda M A and Burguete J 2009 Experimentally observed route to spatiotemporal chaos in an extended one-dimensional array of convective oscillators Phys. Rev. E 79046201

[41] Miranda M A and Burguete J 2010 Spatiotemporal phase synchronization in a large array of convective oscillators Int. J. Bifurcation Chaos 20835

[42] Colombini M and Stocchino A 2008 Finite-amplitude river dunes J. Fluid Mech. 611 283-306 
[43] Ning C-Z and Haken H 1990 Detuned lasers and the complex Lorenz equations: subcritical and supercritical Hopf bifurcations Phys. Rev. A 41 3826-37

[44] Holzner R, Derighetti B, Ravani M and Brun E 1987 Observation of a subcritical Hopf bifurcation in a laser with an injected signal Phys. Rev. A 36 1280-7

[45] Clerc M G, Coulibaly S and Laroze D 2012 Localized waves in a parametrically driven magnetic nanowire Europhys. Lett. 9730006

[46] Clerc M G, Coulibaly S and Laroze D 2008 Localized states beyond the asymptotic parametrically driven amplitude equation Phys. Rev. E 77056209

[47] Clerc M G, Coulibaly S and Laroze D 2009 Nonvariational Ising-Bloch transition in parametrically driven systems Int. J. Bifurcation Chaos 19 2717-26

[48] Cross M C and Hohenberg P C 1993 Pattern formation outside of equilibrium Rev. Mod. Phys. 65 851-1112

[49] Deissler R J 1987 Turbulent bursts, spots and slugs in a generalized Ginzburg-Landau equation Phys. Lett. A $120334-40$

[50] Brachet M E, Coullet P and Fauve S 1987 Propagative phase dynamics in temporally intermittent systems Europhys. Lett. 41017

[51] Thual O and Fauve S 1988 Localized structures generated by subcritical instabilities J. Physique 49 1829-33

[52] Fauve S and Thual O 1990 Solitary waves generated by subcritical instabilities in dissipative systems Phys. Rev. Lett. $64282-4$

[53] van Saarloos W and Hohenberg P C 1990 Pulses and fronts in the complex Ginzburg-Landau equation near a subcritical bifurcation Phys. Rev. Lett. 64 749-52

[54] Deissler R J and Brand H R 1990 The effect of nonlinear gradient terms on localized states near a weakly inverted bifurcation Phys. Lett. A 146 252-5

[55] van Saarloos W and Hohenberg P C 1992 Fronts, pulses, sources and sinks in generalized complex Ginzburg-Landau equations Physica D 56 303-67

[56] Deissler R J and Brand H R 1994 Periodic, quasiperiodic and chaotic localized solutions of the quintic complex Ginzburg-Landau equation Phys. Rev. Lett. 72 478-81

[57] Gutiérrez P, Escaff D, Pérez-Oyarzún S and Descalzi O 2009 Moving breathing pulses in the one-dimensional complex cubic-quintic Ginzburg-Landau equation Phys. Rev. E 80037202

[58] Rajantie A 2003 Defect formation in the early universe Contemp. Phys. 44 485-502

[59] Vachaspati T 2006 Kinks and Domain Walls (Cambridge: Cambridge University Press)
[60] Linde A D 1982 A new inflationary universe scenario: a possible solution of the horizon, flatness, homogeneity, isotropy and primordial monopole problems Phys. Lett. B 108 389-93

[61] Guth A H 1981 Inflationary universe: a possible solution to the horizon and flatness problems Phys. Rev. D 23 347-56

[62] Guth A H and Weinberg E J 1980 Cosmological lower bound on the Higgs-boson mass Phys. Rev. Lett. 45 1131-4

[63] Hawking S W and Moss I L 1982 Supercooled phase transitions in the very early universe Phys. Lett. B $11035-8$

[64] Boyanovsky D, de Vega H J and Schwarz D J 2006 Phase transitions in the early and present universe Annu. Rev. Nucl. Part. Sci. 56 441-500

[65] Durrer R 1999 Topological defects in cosmology New Astron. Rev. 43 111-56

[66] Kibble T W B and Pickett G R 2008 Introduction. cosmology meets condensed matter Phil. Trans. R. Soc. A $3662793-802$

[67] Davis A-C and Kibble T W B 2005 Fundamental cosmic strings Contemp. Phys. 46 313-22

[68] Hindmarsh M B and Kibble T W B 1995 Cosmic strings Rep. Prog. Phys. 58477

[69] Jeannerot R, Rocher J and Sakellariadou M 2003 How generic is cosmic string formation in supersymmetric grand unified theories Phys. Rev. D 68103514

[70] Davis A-C, Brax P and van de Bruck C 2008 Brane inflation and defect formation Phil. Trans. R. Soc. A $3662833-42$

[71] Uzunov D I 1993 Introduction to the Theory of Critical Phenomena (Singapore: World Scientific)

[72] Rajantie A 2002 Formation of topological defects in gauge field theories Int. J. Mod. Phys. A 17 1-43

[73] Erneux T, Reiss E L, Holden L J and Georgiou M 1991 Slow passage through bifurcation and limit points. Asymptotic theory and applications Dynamic Bifurcations (Springer Lecture Notes in Mathematics vol 1493) ed E Benoît (Berlin: Springer) pp 14-28

[74] Rivers R J 2001 Zurek-Kibble causality bounds in time-dependent Ginzburg-Landau theory and quantum field theory J. Low Temp. Phys. 124 41-83

[75] Rajantie A 2009 Super-horizon cosmic string correlations Phys. Rev. D 79043515

[76] Ibaceta D and Calzetta E 1999 Counting defects in an instantaneous quench Phys. Rev. E 60 2999-3008

[77] García-Bellido J, Figueroa D G and Sastre A 2008 Gravitational wave background from reheating after hybrid inflation Phys. Rev. D 77043517

[78] Mulryne D, Orani S and Rajantie A 2011 Non-Gaussianity from the hybrid potential Phys. Rev. D 84123527

[79] Linde A D 1984 The inflationary universe Rep. Prog. Phys. 47925 